\title{
A Dipeptidyl Peptidase-4 Inhibitor Suppresses Macrophage Foam Cell Formation in Diabetic $d b / d b$ Mice and Type 2 Diabetes Patients
}

\author{
Michishige Terasaki $\mathbb{D}^{1}{ }^{1}$ Munenori Hiromura, ${ }^{1}$ Yusaku Mori, ${ }^{1}$ Kyoko Kohashi, ${ }^{1}$ \\ Hideki Kushima, ${ }^{1}$ Masakazu Koshibu, ${ }^{1}$ Tomomi Saito, ${ }^{1}$ Hironori Yashima, \\ Takuya Watanabe, ${ }^{2}$ and Tsutomu Hirano (i) $^{1}$ \\ ${ }^{1}$ Department of Medicine, Division of Diabetes, Metabolism, and Endocrinology, Showa University School of Medicine, Tokyo, Japan \\ ${ }^{2}$ Laboratory of Cardiovascular Medicine, Tokyo University of Pharmacy and Life Sciences, Hachioji City, Tokyo, Japan
}

Correspondence should be addressed to Michishige Terasaki; ttmichishige@yahoo.co.jp

and Tsutomu Hirano; hirano@med.showa-u.ac.jp

Received 29 August 2018; Accepted 4 November 2018; Published 9 December 2018

Academic Editor: Ludwik K. Malendowicz

Copyright (c) 2018 Michishige Terasaki et al. This is an open access article distributed under the Creative Commons Attribution License, which permits unrestricted use, distribution, and reproduction in any medium, provided the original work is properly cited.

\begin{abstract}
Dipeptidyl peptidase-4 (DPP-4) inhibitors could have antiatherosclerotic action, in addition to antihyperglycemic roles. Because macrophage foam cells are key components of atherosclerosis, we investigated the effect of the DPP-4 inhibitor teneligliptin on foam cell formation and its related gene expression levels in macrophages extracted from diabetic $d b / d b$ (C57BLKS/J Iar $-+\operatorname{Lepr}^{d b} /+\operatorname{Lepr}^{d b}$ ) mice and type 2 diabetes (T2D) patients ex vivo. We incubated mouse peritoneal macrophages and human monocyte-derived macrophages differentiated by 7 -day culture with oxidized low-density lipoprotein in the presence/absence of teneligliptin $(10 \mathrm{nmol} / \mathrm{L})$ for 18 hours. We observed remarkable suppression of foam cell formation by teneligliptin treatment ex vivo in macrophages isolated from diabetic $d b / d b$ mice (32\%) and T2D patients (38\%); this effect was accompanied by a reduction of CD36 ( $d b / d b$ mice, $43 \%$; T2D patients, $46 \%)$ and acyl-coenzyme A: cholesterol acyltransferase-1 (ACAT-1) gene expression levels ( $d b / d b$ mice, 47\%; T2D patients, 45\%). Molecular mechanisms underlying this effect are associated with downregulation of CD36 and ACAT-1 by teneligliptin. The suppressive effect of a DPP-4 inhibitor on foam cell formation in T2D is conserved across species and is worth studying to elucidate its potential as an intervention for antiatherogenesis in T2D patients.
\end{abstract}

\section{Introduction}

Type 2 diabetes (T2D) is well known to accelerate the clinical course of atherosclerosis, a condition associated with arterial endothelial dysfunction and several metabolic abnormalities. During the early stage of atherosclerosis, subendothelial accumulation of lipid-laden macrophage-derived foam cells occurs. Accumulation of cholesterol esters in macrophages is a hallmark of foam cell formation, which depends on the uptake of oxidized low-density lipoprotein (ox-LDL) via CD36 [1]. It is decreased by the efflux of free cholesterol mediated by ATP-binding cassette transporter (ABC) A1 and ATP-binding cassette subfamily G member 1 (ABCG1)
[1]. To protect cells from toxicity resulting from excessive free cholesterol accumulation, the free cholesterol is esterified to cholesteryl ester by acyl-coenzyme A: cholesterol acyltransferase-1 (ACAT-1) [2]. ACAT-1 thereby contributes to foam cell formation by promoting cholesteryl ester accumulation in macrophages.

Dipeptidyl peptidase-4 (DPP-4) inhibitors have been widely used to lower glucose levels for the treatment of T2D patients. DPP-4 is an enzyme that degrades active glucagonlike peptide-1 (GLP-1) and active glucose-dependent insulinotropic peptide (GIP), incretins that are secreted on food intake. DPP-4 inhibitors act in concert to stimulate insulin secretion, leading to improved glucose levels. Reportedly, in 
addition to their antihyperglycemic roles, DPP-4 inhibitors have antiatherosclerotic actions in various animal models [3-8]. We previously demonstrated in in vivo studies that GLP-1, GIP, and a DPP-4 inhibitor, respectively, prevented the acceleration of atherosclerosis via suppression of foam cell formation regulated by CD36 and ACAT-1 in macrophages isolated from mice $[3,9]$. Although DPP-4 inhibitors can prevent the accumulation of monocyte/macrophage foam cells in the atherosclerotic plaque formation in animals, their precise effects on macrophage foam cell formation in diabetic rodents and T2D patients remain unclear, especially in ex vivo studies. Therefore, the present study is aimed at elucidating the potential effect of a DPP-4 inhibitor on cardiovascular risk markers, foam cell formation in macrophages, and associated gene expression levels in macrophages isolated from diabetic $d b / d b$ mice and T2D patients ex vivo.

\section{Materials and Methods}

2.1. Animal Experiments. This study was conducted in strict accordance with the recommendations in the Guide for the Care and Use of Laboratory Animals of the National Institutes of Health. The protocol was approved by the Institutional Animal Care and Use Committee of Showa University (permit number: 07005). All surgeries and sacrifices were performed under general anesthesia using isoflurane and with efforts to minimize suffering. Seven 7-week-old male $d b / d b\left(C 57 B L K S / J\right.$ Iar $\left.-+L e p r^{d b} /+L e p r^{d b}\right)$ mice, a mouse model of $\mathrm{T} 2 \mathrm{D}$, and seven 7 -week-old male $d b / d b$ misty $(C 57 B L K S / J$ Iar $-m+/ m+)$ mice were purchased from Sankyo Labo Service (Tokyo, Japan) and kept on standard rodent chow. At 13 weeks of age, peritoneal macrophages and blood samples were collected, as previously described $[3,4,9]$. In brief, after intraperitoneal injection of thioglycolate broth, the peritoneal cells were isolated. The cells were seeded onto $3.5 \mathrm{~cm}$ dishes $\left(3 \times 10^{6}\right.$ cells/dish $)$ and allowed to adhere to the dish, followed by incubation for 1 hour at $37^{\circ} \mathrm{C}$ in $5 \% \mathrm{CO}_{2}$ in RPMI 1640 medium containing 5\% fetal bovine serum (FBS), $100 \mathrm{U} / \mathrm{mL}$ streptomycin, and $100 \mathrm{U} / \mathrm{mL}$ penicillin. After the completion of the incubation, the adherent cells that were identified as peritoneal macrophages were used for a cholesterol esterification assay, reverse transcription polymerase chain reaction (RT-PCR), or immunohistochemistry.

2.2. Measurement of Mouse Plasma. The blood samples collected after a 12-hour fast were used for analysis. Fasting blood glucose (FBG) levels were measured using a Stat strip XP2 dextrometer (Nipro, Osaka, Japan). Hemoglobin A1c (HbA1c) levels were measured using an A1CNow Plus kit (Bayer, Frankfurt, Germany) before sacrificing the animals. Plasma levels of total cholesterol (Total-C), high-density lipoprotein cholesterol (HDL-C), and triglyceride were measured by enzymatic methods (Wako, Osaka, Japan). Plasma levels of insulin were determined by enzyme-linked immunosorbent assay (ELISA) using an ultrasensitive mouse insulin ELISA kit (Morinaga, Kanagawa, Japan). In addition, systolic and diastolic blood pressures (SBP and DBP, respectively) were measured on the day of sacrifice in the fasting state by the tail-cuff method (Model MK-2000ST, Muromachi Kikai, Tokyo, Japan) [3, 4, 9]. In a subset of animals, oral glucose tolerance tests (OGTTs) were conducted, as previously described $[4,10]$.

2.3. Immunofluorescent Staining of Peritoneal Macrophages in Mice. For immunohistochemistry, an anti-F4/80 antibody (Alexa Fluor ${ }^{\circledR}$ 647, Ab204467; Abcam, Cambridge, England) at a concentration of $1: 250$ was added to RPMI 1640 medium with $5 \%$ FBS, which contained $10 \mu \mathrm{g} / \mathrm{mL}$ Dil-oxLDL (Highland Technology Center, MD, USA) with/without $10 \mathrm{nmol} / \mathrm{L}$ teneligliptin [11], and the incubation was continued for 18 hours at $37^{\circ} \mathrm{C}$ in $5 \% \mathrm{CO}_{2}$. After washing, the stained macrophages were mounted in VECTASHIELD HardSet Mounting Medium with DAPI (H-1500, Vector Laboratories, CA, USA) and imaged with a BZ-X710 microscope/software (Keyence, Osaka, Japan).

2.4. Human Experiments. The study protocol was approved by the Ethics Committee of the Showa University School of Medicine (Tokyo, Japan; approval number: 956). Written informed consent was obtained from all T2D patients who had attended at Showa University Hospital and healthy volunteers after receiving a clear explanation of the study protocol. The study was designed in compliance with the Declaration of Helsinki.

The patients were diagnosed with T2D as their blood glucose control specified in the Treatment Guide for Diabetes (Japan Diabetes Society 2010) was inadequately controlled (HbA1c $\geq 7.0 \%$ ) according to the National Glycohemoglobin Standardization Program (NGSP), despite insulin therapy in addition to dietary/exercise therapy or concomitant oral drug therapy other than DPP- 4 inhibitors over $\geq 12$ weeks. The healthy volunteers were free of diabetes mellitus and were taking no medications for diabetes.

After a 12-hour overnight fast, blood samples were collected from four 38-81-year-old male T2D patients and four 36-64-year-old healthy male volunteers. Human peripheral mononuclear cells were isolated from their blood. Monocytes purified using anti-CD14 antibody-conjugated magnetic microbeads (Miltenyi Biotec, CA, USA) were seeded onto $3.5 \mathrm{~cm}$ dishes $\left(1 \times 10^{6}\right.$ cells/dish $)$. The adherent monocytes were incubated at $37^{\circ} \mathrm{C}$ in $5 \% \mathrm{CO}_{2}$ for 7 days in RPMI 1640 medium supplemented with $10 \%$ pooled human serum (HS), $100 \mathrm{U} / \mathrm{mL}$ streptomycin, and $100 \mathrm{U} / \mathrm{mL}$ penicillin to induce differentiation to macrophages, which were subsequently used for the cholesterol esterification assay or RT-PCR.

2.5. Measurement of Characteristics and Biochemical Parameters of the Human Subjects. We used the body mass index (BMI; the weight in kilograms divided by the square of the height in meters) as an index of obesity. SBP and DBP were measured twice with a mercurial sphygmomanometer (YAMASU, KENZMEDICO, Saitama, Japan). FBG, HbA1c (NGSP), LDL-C, HDL-C, and triglyceride were measured by conventional direct methods.

2.6. Cholesterol Esterification Assay in Macrophages Isolated from Mice and Humans. The cholesterol esterification assay 
was conducted as previously described $[3,4,9]$. In brief, mouse peritoneal macrophages or human macrophages differentiated by 7 -day culture were incubated at $37^{\circ} \mathrm{C}$ in $5 \%$ $\mathrm{CO}_{2}$ for 18 hours in RPMI 1640 medium containing $10 \mu \mathrm{g} / \mathrm{mL}$ ox-LDL with $0.1 \mathrm{mmol} / \mathrm{L}\left[{ }^{3} \mathrm{H}\right]$ oleate in the presence/absence of $10 \mathrm{nmol} / \mathrm{L}$ teneligliptin. The cellular lipids were extracted, and the radioactivity of the cholesterol $\left[{ }^{3} \mathrm{H}\right]$ oleate was determined by thin-layer chromatography.

2.7. Gene Expression Levels in Macrophages Isolated from Mice and Humans. Adherent macrophages were incubated at $37^{\circ} \mathrm{C}$ in $5 \% \mathrm{CO}_{2}$ for 18 hours with RPMI 1640 medium with/without $10 \mathrm{nmol} / \mathrm{L}$ teneligliptin ex vivo. Total RNA was isolated from mouse peritoneal macrophages or human macrophages differentiated by 7-day culture using a QIAGEN reagent (Hilden, Germany). Gene expression was assessed by real-time RT-PCR using the TaqMan Gene Expression Assay and Sequence Detection System (ABI PRISM 7900, Life Technologies, CA, USA), as previously described $[9,10]$.

2.8. Statistical Analysis. Values are expressed as mean \pm SEM. Statistical analyses were performed by unpaired $t$-test when two groups were analyzed and ANOVA with Tukey's post hoc test when more than two groups were analyzed. Categorical variables were compared by the chi-square test. All analyses were performed by GraphPad Prism version 7.0 software (GraphPad Software, CA, USA). The significance level was defined as $p<0.05$.

\section{Results}

3.1. Characteristics and Laboratory Data of Mice and Humans. Table 1 shows the characteristics and laboratory data of the diabetic $d b / d b$ mice and $d b / d b$ misty mice. Compared with the $d b / d b$ misty mice, these diabetic $d b / d b$ mice exhibited the classical features of the mouse T2D model, such as severe hyperglycemia (HbAlc, $8.3 \pm 0.2 \%$ vs. $4.2 \pm 0.1 \%$; FBG, $432 \pm 26 \mathrm{mg} / \mathrm{dL}$ vs. $107 \pm 5 \mathrm{mg} / \mathrm{dL}$ ), hyperphagia (food intake, $4.5 \pm 0.2 \mathrm{~g} /$ day vs. $3.1 \pm 0.1 \mathrm{~g} /$ day), high body weight gain ( $43.8 \pm 0.6 \mathrm{~g}$ vs. $25.4 \pm 0.3 \mathrm{~g}$ ), high insulin concentration $(2.48 \pm 0.32 \mathrm{ng} / \mathrm{dL}$ vs. $0.27 \pm 0.03 \mathrm{ng} / \mathrm{dL})$, and high triglyceride levels $(134 \pm 16 \mathrm{mg} / \mathrm{dL}$ vs. $91 \pm 3 \mathrm{mg} / \mathrm{dL})$. Although Total-C and HDL-C levels were slightly higher in the $d b / d b$ mice than in the $d b / d b$ misty mice, the differences were not significant. Consistent with the high FBG and HbAlc levels, the diabetic $d b / d b$ mice showed higher glucose loading after OGTT and area under the curve than the db/db misty mice $(1215 \pm 85 \mathrm{mg} / \mathrm{dL} \times$ hour vs. $276 \pm 3 \mathrm{mg} / \mathrm{dL} \times$ hour$)$.

Table 2 summarizes the characteristics and plasma biochemical parameters of the four T2D patients and four healthy volunteers. In spite of insulin therapy in addition to dietary/exercise therapy or concomitant oral drug therapy other than DPP-4 inhibitors over $\geq 12$ weeks, the T2D patients had high glucose levels than the healthy volunteers (HbA1c, $8.3 \pm 0.2 \%$ vs. $5.4 \pm 0.2 \%$; FBG, $189 \pm 24 \mathrm{mg} / \mathrm{dL}$ vs. $93 \pm 2 \mathrm{mg} / \mathrm{dL}$ ), and the duration of T2D was $19 \pm 6$ years. The T2D patients had progressive diabetic complications such as retinopathy, nephropathy, coronary artery disease, and peripheral artery disease. The use of oral antidiabetic, lipid-lowering, and antihypertensive drugs and the dose of insulin were described. Supplementary Table 1 lists the parameters of the individual T2D patients and healthy volunteers.

3.2. Coexpression of F4/80 and Dil-ox-LDL in Mouse Peritoneal Macrophages. We evaluated whether F4/80 and Dil-ox-LDL were expressed in mouse peritoneal macrophages. Immunofluorescent staining showed that F4/80 and Dil-ox-LDL were mainly coexpressed in the cytoplasm of the mouse peritoneal macrophages (Figures 1(a)-1(i)), suggesting that ox-LDL accumulates in mouse macrophages. The Dil-ox-LDL relative fluorescence ratio per area increased in the diabetic $d b / d b$ mice by 1.54 times compared with that in the $d b / d b$ misty mice, which was reduced by $18 \%$ by ex vivo treatment with teneligliptin in the diabetic $d b / d b$ mice (Figure 1(j)).

3.3. Cholesterol Ester Accumulation in Macrophages Isolated from Mice and Humans. To determine whether teneligliptin regulated foam cell formation in macrophages ex vivo, we assessed the effect of teneligliptin on ox-LDL-induced foam cell formation in the peritoneal macrophages of the diabetic $d b / d b$ mice and monocyte-derived macrophages differentiated by 7 -day culture isolated from the T2D patients. The number of exudate peritoneal cells and morphological cell characteristics did not significantly differ among the groups. ox-LDL-induced cholesterol ester accumulation, which indicated the extent of foam cell formation, was approximately four times higher in the macrophages isolated from the diabetic $d b / d b$ mice than in those from the $d b /$ $d b$ misty mice. This enhanced foam cell formation in the diabetic $d b / d b$ mice was significantly suppressed by $32 \%$ by ex vivo treatment with teneligliptin (Figure $1(\mathrm{k})$ ). On the other hand, in humans, the extent of macrophage foam cell formation in the T2D patients was approximately three times higher than that in healthy volunteers. Interestingly, we observed a remarkable decrease of $38 \%$ in the extent of macrophage foam cell formation by ex vivo treatment with teneligliptin in T2D patients (Figure 2(a)). In addition, we showed the extent of macrophage foam cell formation in individual healthy volunteers (Supplementary Figure 1, ad), T2D patients (Supplementary Figure 1, e-h), and T2D patients with ex vivo teneligliptin treatment after isolation (Supplementary Figure 1, e'-h'). We extracted 4-5 dishes of adhered monocyte-derived macrophages in each group (Supplementary Figure 1, a-h'). The background characteristics and plasma biochemical parameters of individual T2D patients and healthy volunteers are also listed (Supplementary Table 1, a-h).

3.4. Changes in Gene Expression Associated with Macrophage Foam Cell Formation in Mice and Humans. To elucidate the molecular mechanisms underlying the reductive effect of teneligliptin on foam cell formation in macrophages isolated from the diabetic $d b / d b$ mice and T2D patients, we examined gene expression levels associated with foam cell formation in macrophages. CD36 and ACAT-1 gene expression levels in 
TABLE 1: Characteristics and laboratory data of $d b / d b$ misty mice and $d b / d b$ mice at 13 weeks old.

\begin{tabular}{lccc}
\hline & $d b / d b$ misty & $d b / d b$ & $p$ value \\
\hline Number & 7 & 7 & - \\
Food intake (g/day) & $3.1 \pm 0.1$ & $4.5 \pm 0.2$ & $p<0.005^{\star}$ \\
Final body weight (g) & $25.4 \pm 0.3$ & $43.8 \pm 0.6$ & $0.0001^{\star}$ \\
SBP (mmHg) & $106 \pm 4$ & $109 \pm 4$ & 0.622 \\
DBP (mmHg) & $57 \pm 3$ & $61 \pm 2$ & 0.274 \\
Total-C (mg/dL) & $102 \pm 3$ & $112 \pm 16$ & 0.473 \\
HDL-C (mg/dL) & $56 \pm 3$ & $63 \pm 13$ & 0.573 \\
Triglyceride (mg/dL) & $91 \pm 3$ & $134 \pm 16$ & $p<0.05^{\star}$ \\
Insulin (ng/mL) & $0.27 \pm 0.03$ & $2.48 \pm 0.32$ & $p<0.0001^{\star}$ \\
FBG (mg/dL) & $107 \pm 5$ & $432 \pm 26$ & $p<0.0001^{\star}$ \\
HbAlc (\%) & $4.2 \pm 0.1$ & $8.3 \pm 0.2$ & $p<0.0001^{\star}$ \\
OGTT-AUC (mg/dL $\times$ hour) & $276 \pm 3$ & $1215 \pm 85$ & \\
\hline
\end{tabular}

SBP: systolic blood pressure; DBP: diastolic blood pressure; Total-C: total cholesterol; HDL-C: high-density lipoprotein cholesterol; FBG: fasting blood glucose; HbAlc: hemoglobin A1c; OGTT: oral glucose tolerance test; AUC: area under the curve of glucose. Results are presented as mean values \pm SEM and analyzed with unpaired $t$-test. ${ }^{\star} p<0.05$ vs. $d b / d b$ misty mice.

TABLE 2: Characteristics and plasma biochemical parameters of T2D patients and healthy volunteers.

\begin{tabular}{|c|c|c|c|}
\hline & Healthy volunteers & T2D patients & $p$ value \\
\hline Number & 4 & 4 & - \\
\hline Age (years) & $51 \pm 7$ & $63 \pm 9$ & 0.329 \\
\hline Duration of diabetes (years) & - & $19 \pm 6$ & - \\
\hline Body weight (kg) & $69 \pm 3$ & $74 \pm 9$ & 0.666 \\
\hline BMI $\left(\mathrm{kg} / \mathrm{m}^{2}\right)$ & $22.6 \pm 0.8$ & $26.4 \pm 3.2$ & 0.282 \\
\hline $\mathrm{SBP}(\mathrm{mmHg})$ & $117 \pm 3$ & $113 \pm 2$ & 0.376 \\
\hline DBP $(\mathrm{mmHg})$ & $76 \pm 5$ & $70 \pm 3$ & 0.284 \\
\hline Total-C (mg/dL) & $189 \pm 6$ & $187 \pm 10$ & 0.84 \\
\hline LDL-C (mg/dL) & $120 \pm 4$ & $119 \pm 9$ & 0.888 \\
\hline HDL-C (mg/dL) & $45 \pm 6$ & $39 \pm 5$ & 0.393 \\
\hline Triglyceride (mg/dL) & $118 \pm 13$ & $149 \pm 4$ & 0.06 \\
\hline $\mathrm{FBG}(\mathrm{mg} / \mathrm{dL})$ & $93 \pm 2$ & $189 \pm 24$ & $p<0.01^{\star}$ \\
\hline HbA1c (\%) & $5.4 \pm 0.2$ & $8.3 \pm 0.2$ & $p<0.0005^{\star}$ \\
\hline Retinopathy (NDR/SDR/PPDR/PDR) & NA & $2 / 0 / 0 / 2$ & - \\
\hline Nephropathy $(1 / 2 / 3 / 4 / 5)$ & NA & $0 / 3 / 1 / 0 / 0$ & - \\
\hline Macroangiopathy (CVD/CAD/PAD/CAD + PAD/none) & NA & $0 / 0 / 0 / 2 / 2$ & - \\
\hline Daily bolus insulin dose (unit) & - & $36 \pm 9$ & - \\
\hline Daily basal insulin dose (unit) & - & $12 \pm 3$ & - \\
\hline Total daily insulin dose (unit) & - & $48 \pm 12$ & - \\
\hline Use of OADs (Met/Met + Pio/none) & $0 / 0 / 4$ & $1 / 1 / 2$ & 0.264 \\
\hline Lipid-lowering drugs (statin/none) & $2 / 2$ & $4 / 0$ & 0.103 \\
\hline Antihypertensive drugs (ARB/ARB $+\beta$-blocker/none) & $0 / 0 / 4$ & $2 / 1 / 1$ & 0.091 \\
\hline
\end{tabular}

T2D: type 2 diabetes; BMI: body mass index; SBP: systolic blood pressure; DBP: diastolic blood pressure; Total-C: total cholesterol; LDL-C: low-density lipoprotein cholesterol; HDL-C: high-density lipoprotein cholesterol; FBG: fasting blood glucose; HbA1c: hemoglobin A1c; NDR: no diabetic retinopathy; SDR: simple diabetic retinopathy; PPDR: preproliferative diabetic retinopathy; PDR: proliferative diabetic retinopathy; NA: not available; -: none; CVD: cerebrovascular disease; CAD: coronary artery disease; PAD: peripheral artery disease; OADs: oral antidiabetic drugs; Met: metformin; Pio: pioglitazone; ARB: angiotensin II receptor blocker. Results are presented as mean values \pm SEM and analyzed with unpaired $t$-test. Categorical variables were compared by the chi-square test. ${ }^{\star} p<0.05$ vs. healthy volunteers.

macrophages isolated from the diabetic $d b / d b$ mice and T2D patients were approximately 2-3 times higher than those from the $d b / d b$ misty mice and healthy controls, respectively.
Ex vivo teneligliptin treatment lowered the gene expression levels of CD36 ( $d b / d b$ mice, $43 \%$; T2D patients, $46 \%$ ) and ACAT-1 ( $d b / d b$ mice, $47 \%$; T2D patients, $45 \%)$. In addition, 


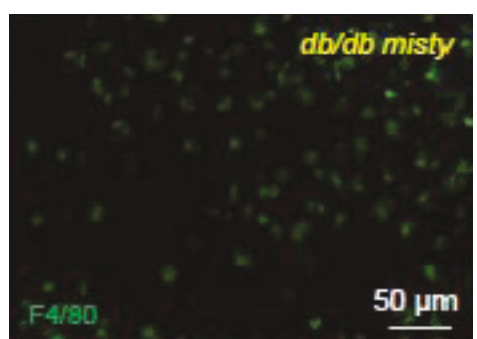

(a)

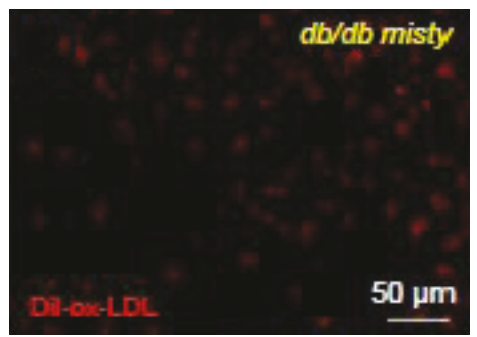

(d)

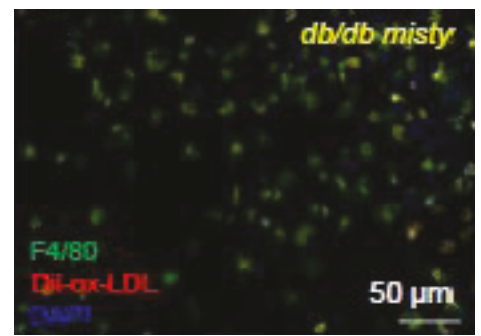

(g)

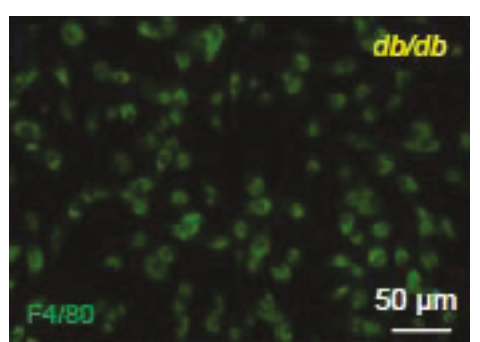

(b)

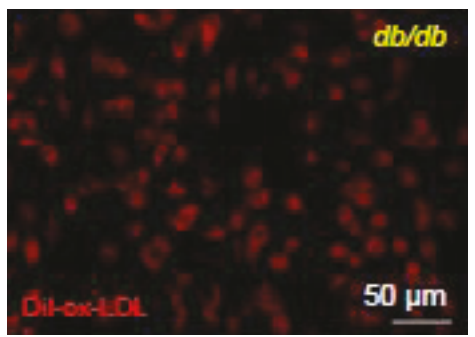

(e)

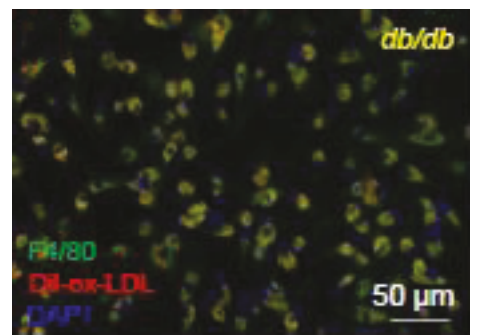

(h)

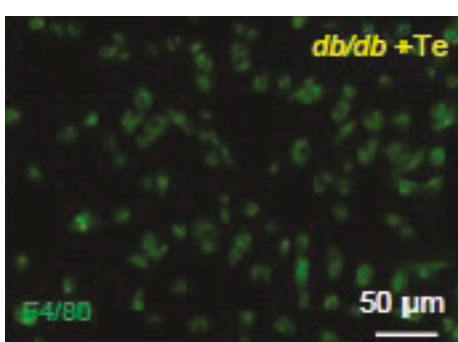

(c)

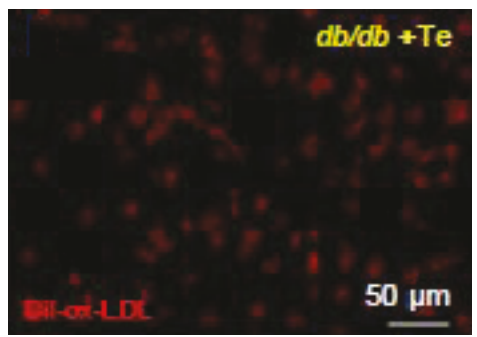

(f)

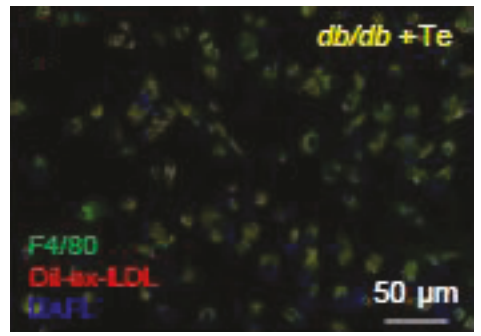

(i)
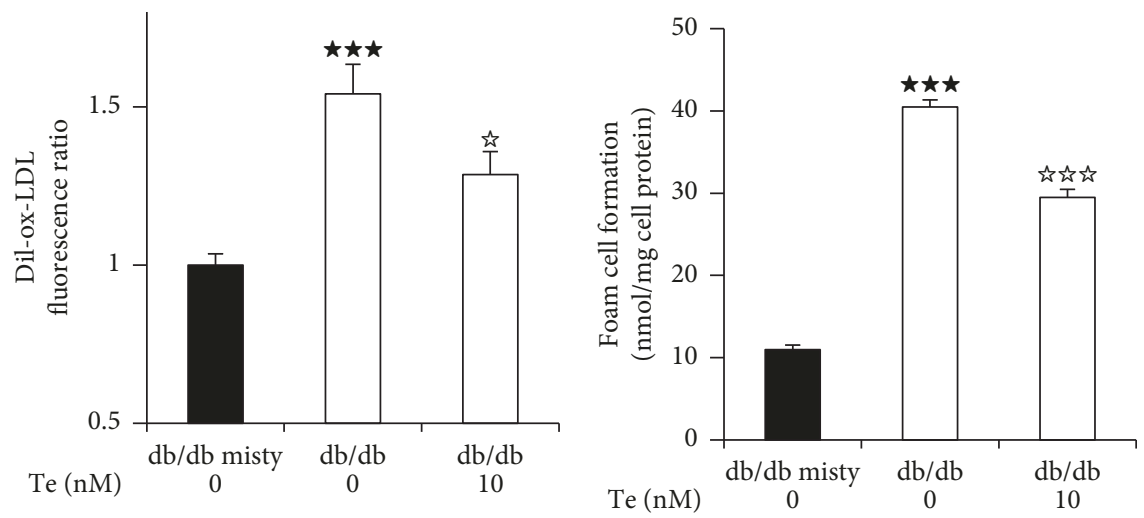

(j)

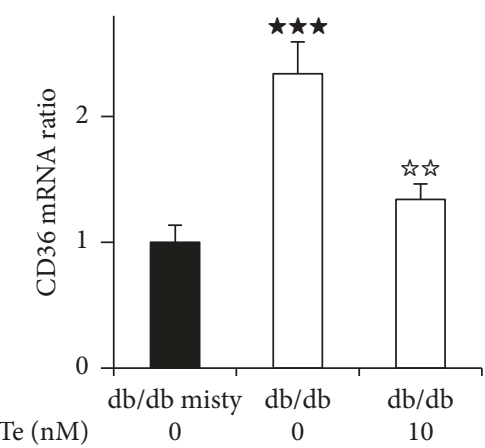

(1)

(k)

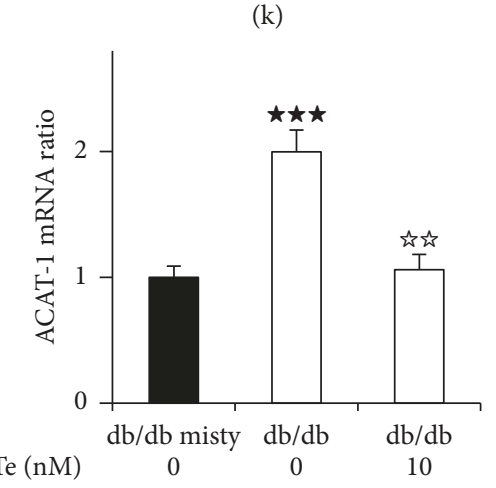

(m)

Figure 1: Continued. 


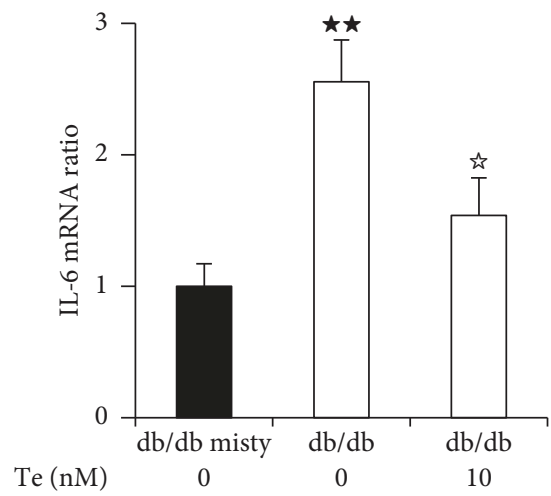

(n)

FIGURE 1: Effects of the dipeptidyl peptidase-4 (DPP-4) inhibitor teneligliptin on oxidized low-density lipoprotein accumulation and its related gene expression levels in macrophages extracted from diabetic $d b / d b$ mice and $d b / d b$ misty mice at 13 weeks old. Four days after an intraperitoneal injection of thioglycolate, the exudate peritoneal cells were isolated from diabetic $d b / d b\left(C 57 B L K S / J\right.$ Iar $-+L e p r^{d b} /$ + Lepr $\left.^{d b}\right)$ mice and $d b / d b$ misty $(C 57 B L K S / J$ Iar $-m+/ m+)$ mice at 13 weeks of age. For cholesterol accumulation, adherent macrophages were incubated at $37^{\circ} \mathrm{C}$ in $5 \% \mathrm{CO}_{2}$ for 18 hours with RPMI 1640 medium containing 5\% fetal bovine serum (FBS) and $10 \mu \mathrm{g} / \mathrm{mL}$ oxidized low-density lipoprotein (ox-LDL) in the presence of $0.1 \mathrm{mmol} / \mathrm{L}\left[{ }^{3} \mathrm{H}\right]$ oleate or $10 \mu \mathrm{g} / \mathrm{mL}$ Dil-ox-LDL, which were added with/without $10 \mathrm{nmol} / \mathrm{L}$ teneligliptin (Te) ex vivo (a-k). For gene expression levels, adherent macrophages were incubated at $37^{\circ} \mathrm{C}$ in $5 \% \mathrm{CO}_{2}$ for 18 hours with RPMI 1640 medium with 5\% FBS in the presence/absence $10 \mathrm{nmol} / \mathrm{L}$ teneligliptin ex vivo without the addition of ox-LDL (l-n). (a-i) Representative immunofluorescent staining images of the peritoneal macrophages isolated from diabetic $d b / d b$ mice or $d b / d b$ misty mice. F4/80-expressing cells were in green, Dil-ox-LDL-stained cells were in red, and DAPI-stained cells were in blue. Scale bars represent $50 \mu \mathrm{m}$. (j) Relative fluorescence ratio per area of Dil-ox-LDL in peritoneal macrophages isolated from mice was imaged and analyzed with a BZ-X710 microscope/software. (k) Foam cell formation in exudate peritoneal macrophages isolated from diabetic $d b / d b$ mice and $d b / d b$ misty mice. The cellular lipids were extracted, and the radioactivity of the cholesterol $\left[{ }^{3} \mathrm{H}\right]$ oleate was determined by thin-layer chromatography. (l-n) Gene expression levels related to foam cell formation in peritoneal macrophages isolated from diabetic $d b / d b$ mice and $d b / d b$ misty mice. Gene expression levels of CD36 (1), acyl-coenzyme A: cholesterol acyltransferase-1 (ACAT-1) (m), and interleukin-6 (IL-6) (n) and the association with GAPDH were analyzed by real-time RT-PCR without the addition of ox-LDL. Data information: $n=7 d b / d b$ misty mice, $n=7 d b / d b$ mice, and $n=7 d b / d b$ mice with ex vivo teneligliptin treatment. Results are presented as mean values \pm SEM and analyzed with one-way ANOVA: ${ }^{\star \star \star} p<0.005,{ }^{\star \star} p<0.01$ vs. db/db misty mice. ${ }^{2}$ i 2 s $p<0.005$, 到 $p<0.01$, $p<0.05$ vs. $d b / d b$ mice without teneligliptin.

the gene expression levels of the proinflammatory cytokine interleukin-6 (IL-6) in macrophages showed similar changes ( $d b / d b$ mice, $42 \%$; T2D patients, 33\%) (Figures 1(l)-1(n) and 2(b) $-2(d))$.

\section{Discussion}

To the best of our knowledge, the present ex vivo study is the first to demonstrate the suppressive effect of a DPP-4 inhibitor on foam cell formation in macrophages isolated from diabetic $d b / d b$ mice and T2D patients. This study showed that the extent of macrophage foam cell formation in diabetic mice and T2D patients significantly increased compared with that in nondiabetic mice and healthy volunteers, which was accompanied by enhanced CD36 and ACAT-1 expression levels. There have been several supportive studies showing that CD36 and ACAT-1 expression or protein was highly expressed in monocytes/macrophages from diabetic mice and T2D patients [12-14]. The exact mechanism of hyperglycemia-induced enhancement of CD36 and ACAT1 remains unknown; however, hyperglycemia-mediated accumulation of reactive oxygen species has the possibility to induce proinflammatory signaling, including p38 mitogen-activated protein kinase, leading to the induction of the nuclear transcription factor-kappaB (NF- $\kappa$ B) signaling pathway and subsequent increase in CD36 expression [15].
Another key factor, ACAT-1, a rate-limiting enzyme for the esterification of free cholesterol from ox-LDL, plays a crucial role in foam cell formation in macrophages characterized by cholesterol ester accumulation [16]. Because ACAT-1 expression is regulated by free cholesterol availability inside cells [2], the changes of ACAT-1 expression levels might be a consequence of changes in CD36 levels.

We previously showed in in vivo studies that CD36 and ACAT- 1 levels in mouse macrophages were decreased by the treatment with GLP-1, GIP, or DPP-4 inhibitors $[3,9]$. In addition, because diabetic $d b / d b$ mice do not accelerate visible atherosclerotic lesions, macrophage foam cell formation was used as a surrogate marker for atherosclerosis in our previous studies $[4,10]$. Dai et al. reported that DPP-4 inhibitors directly repress foam cell formation in THP-1 macrophages by inhibiting the scavenger receptor CD36 in vitro [17], indicating that DPP-4 inhibitors directly suppress macrophage foam cell formation, independent of increased incretins. However, this effect of DPP-4 inhibitors on T2D patients and diabetic mice has not been clarified in an in vitro study. In the present study, we observed remarkable suppression of foam cell formation by ex vivo treatment with a DPP-4 inhibitor in macrophages isolated from diabetic $d b / d b$ mice and T2D patients; this effect was accompanied by a reduction of CD36 and ACAT-1 expression levels. Thus, our findings here are the first to show that a DPP-4 


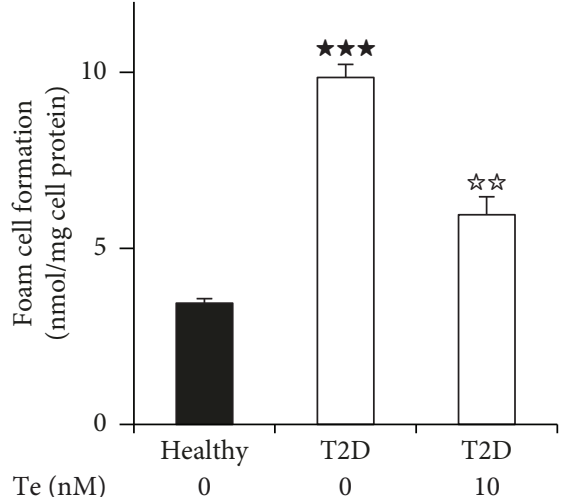

(a)

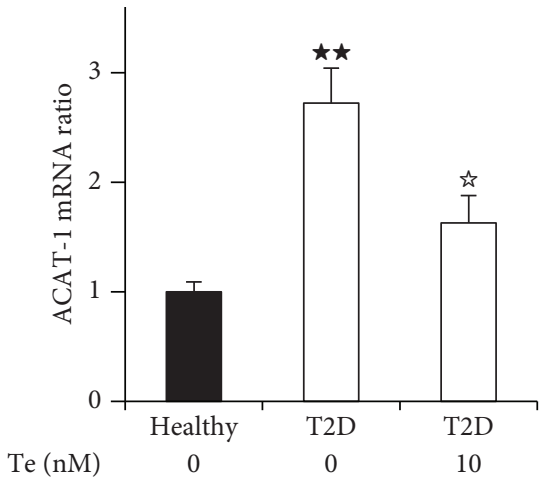

(c)

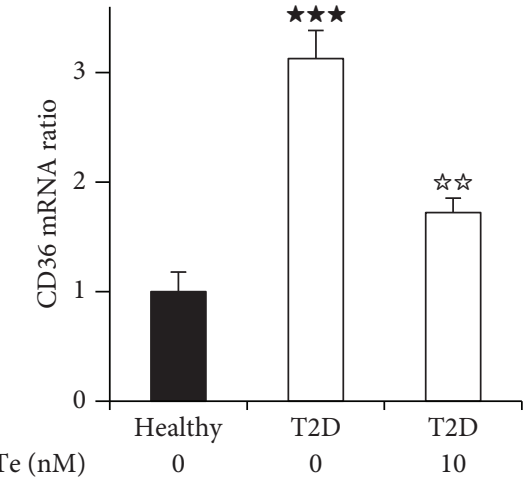

(b)

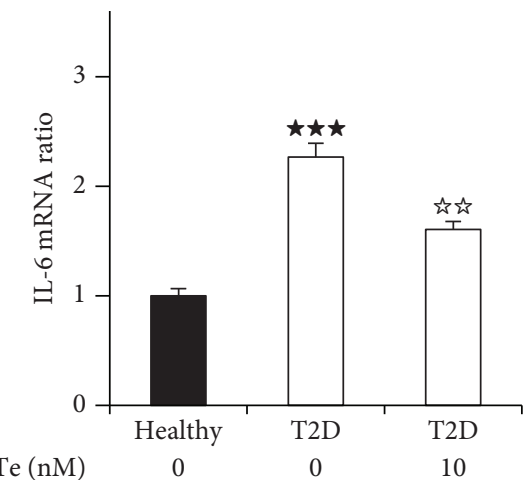

(d)

FIGURE 2: Effects of teneligliptin on foam cell formation and its related gene expression levels in monocyte-derived macrophages isolated from type 2 diabetes (T2D) patients and healthy volunteers. Human peripheral mononuclear cells were isolated from four type 2 diabetes (T2D) patients and four healthy volunteers. Monocytes purified using anti-CD14 antibody-conjugated magnetic microbeads were seeded onto dishes. Adherent monocytes were incubated at $37^{\circ} \mathrm{C}$ in $5 \% \mathrm{CO}_{2}$ for 18 hours with RPMI 1640 medium containing $10 \%$ human serum (HS) for 7 days to induce differentiation to macrophages, which were in the presence/absence of $10 \mathrm{nmol} / \mathrm{L}$ teneligliptin. (a) Human macrophages differentiated by 7-day culture were incubated at $37^{\circ} \mathrm{C}$ in $5 \% \mathrm{CO}_{2}$ for 18 hours in RPMI 1640 medium containing $10 \% \mathrm{HS}$ with $10 \mu \mathrm{g} / \mathrm{mL}$ ox-LDL and $0.1 \mathrm{mmol} / \mathrm{L}\left[{ }^{3} \mathrm{H}\right]$ oleate in the presence/absence of $10 \mathrm{nmol} / \mathrm{L}$ teneligliptin ex vivo. The cellular lipids were extracted, and the radioactivity of the cholesterol $\left[{ }^{3} \mathrm{H}\right]$ oleate was determined by thin-layer chromatography. (b-d) Gene expression levels related to foam cell formation in monocyte-derived macrophages isolated from $\mathrm{T} 2 \mathrm{D}$ patients and healthy volunteers. Adherent macrophages were incubated at $37^{\circ} \mathrm{C}$ in $5 \% \mathrm{CO}_{2}$ for 18 hours with RPMI 1640 medium containing $10 \%$ HS in the presence/absence of $10 \mathrm{nmol} / \mathrm{L}$ teneligliptin ex vivo. Gene expression levels of CD36 (b), ACAT-1 (c), and IL-6 (d) and the association with GAPDH were analyzed by real-time RT-PCR without the addition of ox-LDL. Data information: $n=4$ healthy volunteers, $n=4$ T2D patients, and $n=4$ T2D patients with ex vivo teneligliptin treatment after isolation. Results are presented as mean values \pm SEM and analyzed with one-way ANOVA: ${ }^{\star \star \star} p<0.005,{ }^{\star \star} p<0.01$ vs. health volunteers. ${ }^{\star \star 2} p<0.01$, ${ }^{\star} p<0.05$ vs. T2D patients without ex vivo teneligliptin treatment.

inhibitor has the potential to suppress foam cell formation regulated by CD36 and ACAT-1 in macrophages extracted from diabetic mice and T2D patients. Because previous studies have shown that CD36 and ACAT-1 levels are mainly associated with PI3K and PKC [17-19], a DPP-4 inhibitor can possibly regulate $\mathrm{CD} 36$ and ACAT-1 expressions via the PI3K/PKC signaling pathway in macrophages of diabetic mice and T2D patients. Future studies should be needed to clarify the complex networks of these unidentified pathways concerned in macrophages.

Recent studies support the crucial role of inflammation in the pathogenesis of atherosclerosis and the strong association between hyperglycemia and systemic inflammation. Because inflammation is another key factor for atherogenesis [20], there have been some in vivo studies of anti-inflammatory and antiatherosclerotic effects of DPP-4 inhibitors on animal models and T2D patients [8, 21]. Tremblay et al. [21] showed that plasma levels of IL-6 in T2D patients are reduced by a DPP-4 inhibitor. Sato-Asahara et al. [22] observed that serum IL-6 and monocyte IL-6 expression levels in T2D patients tended to decrease due to in vivo treatment with DPP-4 inhibitors. However, these researchers failed to demonstrate the suppressive effect of DPP-4 inhibitors on monocytes/macrophages in T2D patients ex vivo. We previously reported that ex vivo treatment with teneligliptin led to remarkable suppression of lipopolysaccharide-induced IL-6 expression levels in mouse peritoneal macrophages and human monocyte/macrophage U937 cells [11]. Therefore, we selected the proinflammatory cytokine IL-6 in this study to evaluate the effect of a DPP-4 inhibitor on the proinflammatory function of macrophages isolated from diabetic mice and T2D patients. Our findings show that in addition to 
suppressing foam cell formation, ex vivo teneligliptin treatment decreased inflammatory signals in macrophages, which may also provide support for contributing to the protection against atherosclerosis in high inflammatory conditions such as T2D.

The present study has some potential limitations in human experiments. Therapy with insulin and oral antidiabetic, lipid-lowering, and antihypertensive drugs may partly contribute to foam cell formation in monocyte-derived macrophages isolated from T2D patients. Additionally, there were some differences in terms of body weight and lipid parameters between humans and mice in this study. The lack of evaluations of these differences is another limitation in this study. Future studies are needed to clarify these differences with foam cell formation in macrophages. The mouse experiment in this study is subject to some limitations. The protein levels of regulated CD36 and ACAT-1, as well as the activity levels of ACAT-1 and other inflammatory cytokine NF- $\kappa \mathrm{B}$ compounds, were not evaluated. Nevertheless, the extent of foam cell formation in macrophages isolated from diabetic mice and T2D patients was enhanced in comparison with the formation in those from nondiabetic mice and healthy volunteers, respectively. Further, we observed remarkable suppression of macrophage foam cell formation by ex vivo treatment with a DPP-4 inhibitor in T2D patients, which was consistent with the results observed in the diabetic $d b$ / $d b$ mice. Although the number of human subjects might be insufficient in this study, our findings imply that a DPP-4 inhibitor suppresses macrophage foam cell formation on both species.

\section{Conclusions}

The present study is the first to demonstrate that a DPP-4 inhibitor has the potential to suppress foam cell formation via CD36 and ACAT-1 in macrophages isolated from diabetic $d b / d b$ mice and T2D patients ex vivo. This effect is conserved across species and is worth studying further to elucidate its potential as an intervention for antiatherogenesis in T2D patients.

\section{Data Availability}

All data used to support the findings of this study are available from the corresponding author upon request.

\section{Disclosure}

The funder had no role in the design of this study, data collection and analysis, preparation of the manuscript, and decision to publish.

\section{Conflicts of Interest}

The authors declare that there is no conflict of interest.

\section{Authors' Contributions}

MT designed the study, conducted the experiments of mice and humans, analyzed the data, and wrote the manuscript.
$\mathrm{MH}$ conducted the mouse experiments and analyzed the data. YM analyzed the data and contributed to data interpretation. KK, HK, MK, TS, and HY researched and analyzed the data and contributed to the revision of the manuscript. TW contributed to the discussion and review. TH conceived the study and revised and finalized the manuscript. All authors read and approved the final version of the manuscript for submission. Michishige Terasaki and Tsutomu Hirano contributed equally to this work.

\section{Acknowledgments}

The authors would like to thank Kyoko Nohtomi and Hiroko Takeuchi for their technical assistance. This study was supported by research funds from Mitsubishi Tanabe Pharma Co. (to TH).

\section{Supplementary Materials}

Supplementary 1. Supplementary Figure 1: effects of teneligliptin on foam cell formation in monocyte-derived macrophages isolated from individual T2D patients and healthy volunteers. Human peripheral mononuclear cells were isolated from each of four healthy volunteers (a-d) or four T2D patients (e-h). The cellular lipids were extracted, and the radioactivity of the cholesterol $\left[{ }^{3} \mathrm{H}\right]$ oleate was determined by thin-layer chromatography. We extracted 4-5 dishes of adhered monocyte-derived macrophages isolated from each of healthy volunteers (a-d), T2D patients (e-h), and T2D patients with $10 \mathrm{nmol} / \mathrm{L}$ ex vivo teneligliptin treatment after isolation ( $\left.e^{\prime}-h^{\prime}\right)$. The background characteristics and plasma biochemical parameters of individual T2D patients and healthy volunteers are also listed in Supplementary Table 1. Data information: results are presented as mean values \pm SEM and analyzed with one-way ANOVA: ${ }^{\star \star \star} p<0.005$ vs. health volunteers. ${ }^{\text {sis }} p<0.01$ vs. T2D without teneligliptin ex vivo.

Supplementary 2. Supplementary Table 1: individual characteristics and plasma biochemical parameters of T2D patients and healthy volunteers.

\section{References}

[1] S. Allahverdian, P. S. Pannu, and G. A. Francis, "Contribution of monocyte-derived macrophages and smooth muscle cells to arterial foam cell formation," Cardiovascular Research, vol. 95, no. 2, pp. 165-172, 2012.

[2] P. Saha, B. Modarai, J. Humphries et al., "The monocyte/macrophage as a therapeutic target in atherosclerosis," Current Opinion in Pharmacology, vol. 9, no. 2, pp. 109-118, 2009.

[3] M. Terasaki, M. Nagashima, T. Watanabe et al., "Effects of PKF275-055, a dipeptidyl peptidase-4 inhibitor, on the development of atherosclerotic lesions in apolipoprotein E-null mice," Metabolism, vol. 61, no. 7, pp. 974-977, 2012.

[4] M. Terasaki, M. Nagashima, K. Nohtomi et al., "Preventive effect of dipeptidyl peptidase- 4 inhibitor on atherosclerosis is mainly attributable to incretin's actions in nondiabetic and diabetic apolipoprotein E-null mice," PLoS One, vol. 8, no. 8, article e70933, 2013. 
[5] N. N. Ta, C. A. Schuyler, Y. Li, M. F. Lopes-Virella, and Y. Huang, "DPP-4 (CD26) inhibitor alogliptin inhibits atherosclerosis in diabetic apolipoprotein E-deficient mice," Journal of Cardiovascular Pharmacology, vol. 58, no. 2, pp. 157-166, 2011.

[6] J. Matsubara, S. Sugiyama, K. Sugamura et al., "A dipeptidyl peptidase-4 inhibitor, des-fluoro-sitagliptin, improves endothelial function and reduces atherosclerotic lesion formation in apolipoprotein E-deficient mice," Journal of the American College of Cardiology, vol. 59, no. 3, pp. 265-276, 2012.

[7] N. Ervinna, T. Mita, E. Yasunari et al., "Anagliptin, a DPP-4 inhibitor, suppresses proliferation of vascular smooth muscles and monocyte inflammatory reaction and attenuates atherosclerosis in male apo E-deficient mice," Endocrinology, vol. 154, no. 3, pp. 1260-1270, 2013.

[8] T. Hirano, S. Yamashita, M. Takahashi, H. Hashimoto, Y. Mori, and M. Goto, "Anagliptin, a dipeptidyl peptidase-4 inhibitor, decreases macrophage infiltration and suppresses atherosclerosis in aortic and coronary arteries in cholesterolfed rabbits," Metabolism, vol. 65, no. 6, pp. 893-903, 2016.

[9] M. Nagashima, T. Watanabe, M. Terasaki et al., "Native incretins prevent the development of atherosclerotic lesions in apolipoprotein E knockout mice," Diabetologia, vol. 54, no. 10, pp. 2649-2659, 2011.

[10] M. Terasaki, M. Hiromura, Y. Mori et al., "Combination therapy with a sodium-glucose cotransporter 2 inhibitor and a dipeptidyl peptidase- 4 inhibitor additively suppresses macrophage foam cell formation and atherosclerosis in diabetic mice," International Journal of Endocrinology, vol. 2017, Article ID 1365209, 9 pages, 2017.

[11] M. Hiromura, K. Nohtomi, Y. Mori et al., "Caveolin-1, a binding protein of $\mathrm{CD} 26$, is essential for the anti-inflammatory effects of dipeptidyl peptidase-4 inhibitors on human and mouse macrophages," Biochemical and Biophysical Research Communications, vol. 495, no. 1, pp. 223-229, 2018.

[12] E. Griffin, A. Re, N. Hamel et al., "A link between diabetes and atherosclerosis: glucose regulates expression of CD36 at the level of translation," Nature Medicine, vol. 7, no. 7, pp. 840846, 2001.

[13] M. J. Sampson, I. R. Davies, S. Braschi, K. Ivory, and D. A. Hughes, "Increased expression of a scavenger receptor (CD36) in monocytes from subjects with type 2 diabetes," Atherosclerosis, vol. 167, no. 1, pp. 129-134, 2003.

[14] M. Kaplan, R. Kerry, M. Aviram, and T. Hayek, "High glucose concentration increases macrophage cholesterol biosynthesis in diabetes through activation of the sterol regulatory element binding protein 1 (SREBP1): inhibitory effect of insulin," Journal of Cardiovascular Pharmacology, vol. 52, no. 4, pp. 324332, 2008.

[15] H. Lu, K. Yao, D. Huang et al., "High glucose induces upregulation of scavenger receptors and promotes maturation of dendritic cells," Cardiovascular Diabetology, vol. 12, no. 1, p. 80, 2013.

[16] M. Sekiya, J. Osuga, M. Igarashi, H. Okazaki, and S. Ishibashi, "The role of neutral cholesterol ester hydrolysis in macrophage foam cells," Journal of Atherosclerosis and Thrombosis, vol. 18, no. 5, pp. 359-364, 2011.

[17] Y. Dai, X. Wang, Z. Ding, D. Dai, and J. L. Mehta, “DPP-4 inhibitors repress foam cell formation by inhibiting scavenger receptors through protein kinase C pathway," Acta Diabetologica, vol. 51, no. 3, pp. 471-478, 2014.
[18] S. Hongo, T. Watanabe, S. Arita et al., "Leptin modulates ACAT1 expression and cholesterol efflux from human macrophages," American Journal of Physiology. Endocrinology and Metabolism, vol. 297, no. 2, pp. E474-E482, 2009.

[19] T. Watanabe, T. Suguro, T. Kanome et al., "Human urotensin II accelerates foam cell formation in human monocyte-derived macrophages," Hypertension, vol. 46, no. 4, pp. 738-744, 2005.

[20] C. Antoniades, C. Cunnington, A. Antonopoulos et al., "Induction of vascular GTP-cyclohydrolase I and endogenous tetrahydrobiopterin synthesis protect against inflammationinduced endothelial dysfunction in human atherosclerosis," Circulation, vol. 124, no. 17, pp. 1860-1870, 2011.

[21] A. J. Tremblay, B. Lamarche, C. F. Deacon, S. J. Weisnagel, and P. Couture, "Effects of sitagliptin therapy on markers of low-grade inflammation and cell adhesion molecules in patients with type 2 diabetes," Metabolism, vol. 63, no. 9, pp. 1141-1148, 2014.

[22] N. Satoh-Asahara, Y. Sasaki, H. Wada et al., "A dipeptidyl peptidase-4 inhibitor, sitagliptin, exerts anti-inflammatory effects in type 2 diabetic patients," Metabolism, vol. 62, no. 3, pp. 347-351, 2013. 


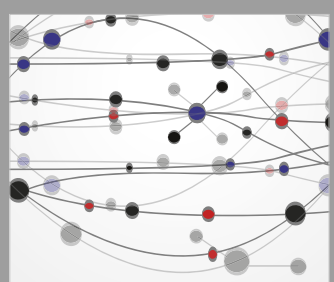

The Scientific World Journal
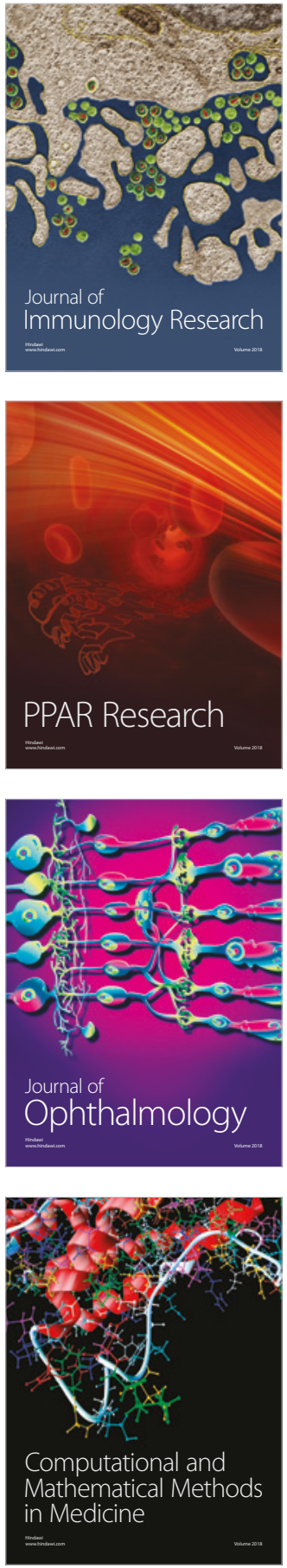

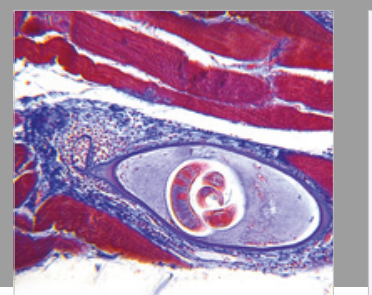

Gastroenterology Research and Practice

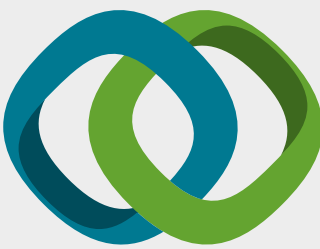

\section{Hindawi}

Submit your manuscripts at

www.hindawi.com
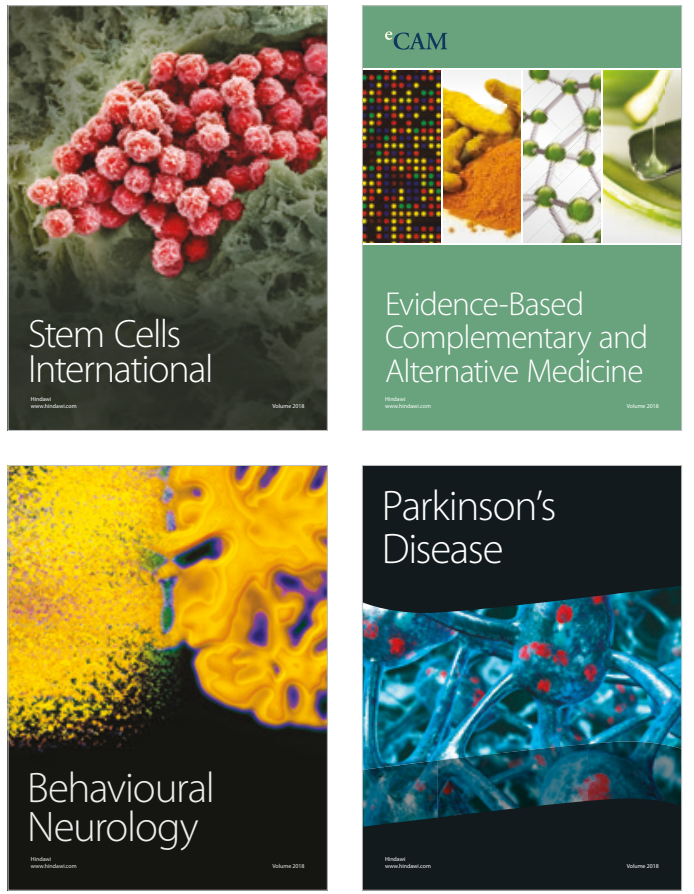

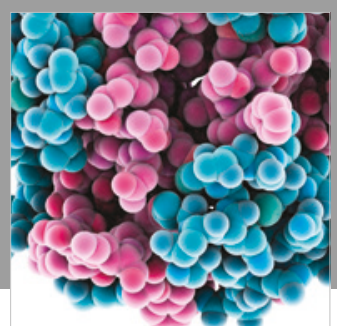

ournal of

Diabetes Research

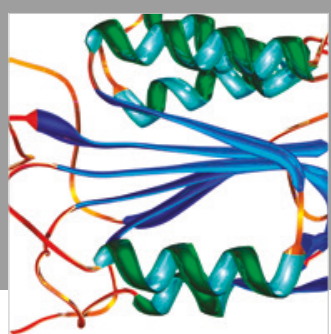

Disease Markers
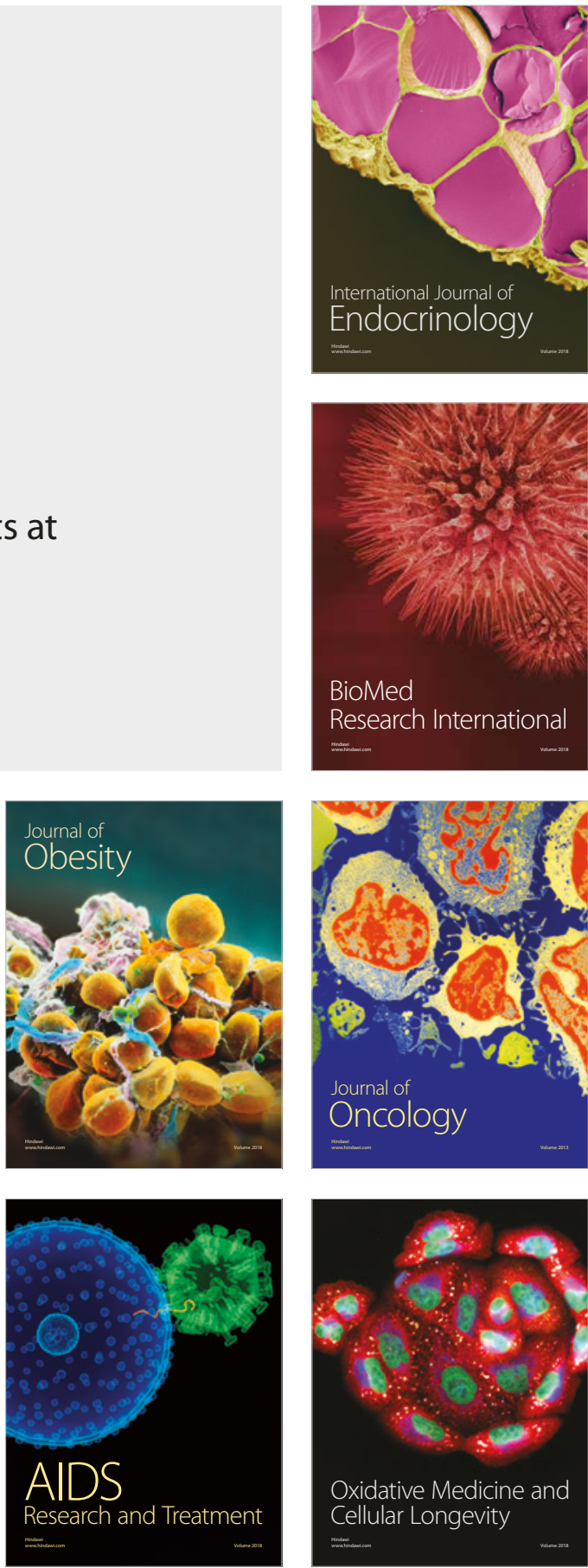